\title{
An Efficient Data Embedding Techniques in Image using Levelling of 2-D DWT and Cryptography
}

\author{
Sheshang Degadwala1, Arpana Mahajan², Dhairya Vyas³, Mouly Purohit ${ }^{4}$ \\ ${ }^{1}$ Head of Department, Computer Engineering, Sigma Institute of Engineering, Vadodara, Gujarat, India \\ ${ }^{2}$ Assistant Professor, Computer Engineering, Sigma Institute of Engineering, Vadodara, Gujarat, India \\ ${ }^{3}$ Assistant Professor, EC Engineering, Sigma Institute of Engineering, Vadodara, Gujarat, India \\ ${ }^{4}$ UG Scholar, Computer Engineering, Sigma Institute of Engineering, Vadodara, Gujarat, India
}

\section{ABSTRACT}

The protecting mystery of delicate information turns out to be essential in today computerized correspondence. Steganography is the train of trading top mystery data by installing it into a mixed media bearer and Cryptography is the specialty of securing data by changing it into an imperceptible frame. A definitive point, here is to shroud the very presence of the installed data inside apparently harmless bearers and transmit such that the presence of data is imperceptible. In this paper, proposed technique removes by joining the highlights of cryptography and steganography. Cryptography utilizing Modified ASCII Conversion and Mathematical Function includes changing over the mystery message into unprintable type of same size as unique message in any cases. Steganography is then connected utilizing multilevel 2-D DWT with LSB to install this encoded information into a cover media utilizing High Frequency Coefficients at all levels of 2-D Haar DWT and shrouds its reality. At long last, Performance can be estimated by utilizing measurable parameters PSNR and MSE. This proposed strategy accomplish each of the three parts of information covering up, for example Capacity, Robustness and Security.

Keywords: Haar DWT, LSB, ASCII Conversion, Cyclic Mathematical Function, PSNR, MSE

\section{INTRODUCTION}

Security of data winds up plainly a standout amongst the most vital components of data innovation and correspondence in light of the gigantic ascent of the World Wide Web and the copyrights laws. Cryptography was begun as a strategy for securing the secrecy of data. Shockingly, it is some of the time insufficient to keep the substance of a message mystery, it might likewise be important to keep the presence of the message mystery and the idea in charge of this is called steganography [2]. Steganography is the act of concealing mystery message inside any media. Most information concealing frameworks exploit human perceptual shortcomings. Steganography is regularly mistaken for cryptography in light of the fact that the two are comparative in the way that they both are utilized to ensure mystery data. In the event that both the strategies: cryptography and steganography is utilized then the correspondence turns out to be twofold secured [2].The fundamental contrast amongst Steganography and cryptography is that, cryptography focuses on keeping the substance of a message mystery while steganography focuses on keeping the presence of a message mystery. ASCII Conversion and cyclic numerical capacity based cryptography is another cryptographic calculation which takes after an alternate approach from the customary symmetric-key cryptography, unbalanced key cryptography or hashing capacity [11]. 
This paper utilizes Modified idea in view of this cryptography calculation for information encryption, where the information will be changed over into an unprintable frame, which will be then covered up into a picture document. So as to empower vast limit of information and keeping up god visual nature of the cover picture, the installing is connected by changing the slanting points of interest coefficients (High recurrence coefficients) in change space of Multilevel Two-Dimensional Haar Discrete Wavelet Transform (HDWT). Minimum huge piece (LSB) inclusion is a typical and straightforward way to deal with install data in a picture document [4]. In this strategy the LSB of a byte is supplanted with a $\mathrm{M}^{\mathrm{ce}} \mathrm{s}$ bit. This procedure functions admirably for picture steganography [4]. The upsides of utilizing this framework is that it doesn't require the first cover picture for fruitful extraction of the mystery message.

\section{METHODOLOGY}

\section{A. Cryptography Approach}

It is another cryptographic calculation which takes after an alternate procedure from the conventional symmetric-key cryptography, deviated key cryptography or hashing capacity. It is utilized to make the scrambled message without a doubt unprintable utilizing a few times of ASCII transformations and a cyclic numerical capacity. Separating the first message into parcels. Twofold lattices are framed for every bundle to create the unprintable scrambled message through influencing the ASCII to an incentive for each character underneath 32. So also, a few ASCII transformations and the reverse cyclic numerical capacity are utilized to unscramble the unprintable encoded message. The last scrambled message got from three times of encryption turns into an unprintable content through which the calculation has larger amount of security without expanding the span of information or loosing of any information when size of unique message is distinguishable or not by Packet estimate.

\section{B. Transform Domain Approach}

Two dimensional (2-D) Discrete wavelet change (DWT) strategy is a standout amongst the most essential systems in changing a spatial area picture into a recurrence space picture. This sort of twodimensional DWT prompts a disintegration of estimate coefficients at level $j$ in four parts: the guess at level $j+1$ and the subtle elements in three introductions (flat, vertical, and corner to corner). HDWT is the least demanding and most ordinarily utilized technique. HDWT can be actualized by two techniques: (1) Horizontal Operation and (2) Vertical Operation. In the first place the Horizontal Operation is used to deteriorate a picture into a low recurrence band (L) and a high recurrence band $(\mathrm{H})$. Second Vertical Operation is used to segment $\mathrm{L}$ and $\mathrm{H}$ into $\mathrm{LL}$, $\mathrm{LH}, \mathrm{HL}$ and $\mathrm{HH}$ distinctive recurrence groups, each of which has $1 / 4$ of the first picture measure. $\mathrm{HH}$ speak to High Frequency band, LL is low recurrence band and LH and HL are center recurrence groups. The coefficients in LL are foremost. In the event that any of the coefficients in LL recurrence band are changed, spectator can noticeably observe that the comparing spatial area picture has been changed. Human eyes are not delicate to change of HDWT coefficients in $\mathrm{HH}$. For any reason, when any coefficients in $\mathrm{HH}$ are adjusted, an eyewitness can exhaustingly (troublesomely) recognize the adjustment in the spatial area picture. After change, store message bits into $\mathrm{HH}$ recurrence coefficients we can utilize LSB technique since it is least difficult and exceptionally well known strategy in spatial space. When utilizing LSB, we needn't bother with unique cover picture for removing mystery message than that are required into different techniques in spatial area, for example, XOR Method.

In multilevel 2-D HDWT, we can apply 2-D HDWT on NXN picture up to $\mathrm{k}$ level where $\mathrm{k}=\log 2 \mathrm{~N}$. For ex, if picture has 256X256 size then we can apply 2-D HDWT on that picture up to $\mathrm{k}=\log 2(\mathrm{~N})=\log 2256=8$ 
levels. How about we consider picture has 8X8 pixel estimate then deterioration of this picture utilizing multilevel 2-D HDWT up to 3-levels appeared in Figure 1
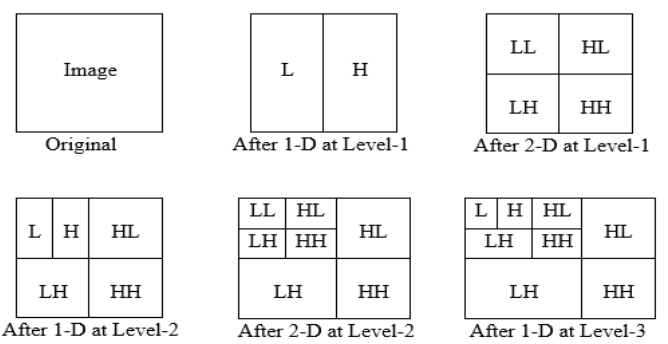

\begin{tabular}{|c|c|c|c|}
\hline LL & HL & HL & \multirow{2}{*}{ HL } \\
\cline { 1 - 2 } LH & HH & & \\
\hline LH & HH & \\
\hline \multicolumn{3}{|c|}{ LH } & HH \\
\hline \multicolumn{3}{|c|}{ After 2-D at Level-3 }
\end{tabular}

Figure 1. 3- Level 2-D HDWT

\section{PROPOSED WORK}

\section{A. Embedding Procedure}

Input: An $\mathrm{n} \times \mathrm{n}$ color image and a secret message.

Output: An $n \times n$ stego-image.

Algorithm:

\section{Steps:}

1. Read the Cover RGB Color image.

2. Separate Each Color Components from Cover Image such as R-Color Image, G-Color Image and B-Color Image.
3. Read the secret message.

4. Apply Encryption procedure of Modified ASCII conversion and Cyclic mathematical function based Cryptography to create binary message vector.

5. Find length of Binary Message Vector and calculate maximum level of HDWT by $K=\log _{2} n$ levels.

6. If length of binary message vector is greater than zero and current level is less than maximum level (K) then apply 2-D Haar Discrete Wavelet Transform at Next Level on each Image Components of Cover Image otherwise go to step 9.

7. Replace Least Significant Bit (LSB) of high Frequency Coefficients of Each Image Components of cover image by secret message bits from message vector and remove those bits from message vector.

8. After storing message bits in all high frequency coefficients of each image components, again calculate length of binary message vector and go to step 6.

9. Combine all Stego image components such as RImage, G-Image and B-Image to prepare Stego RGB Color image for display.

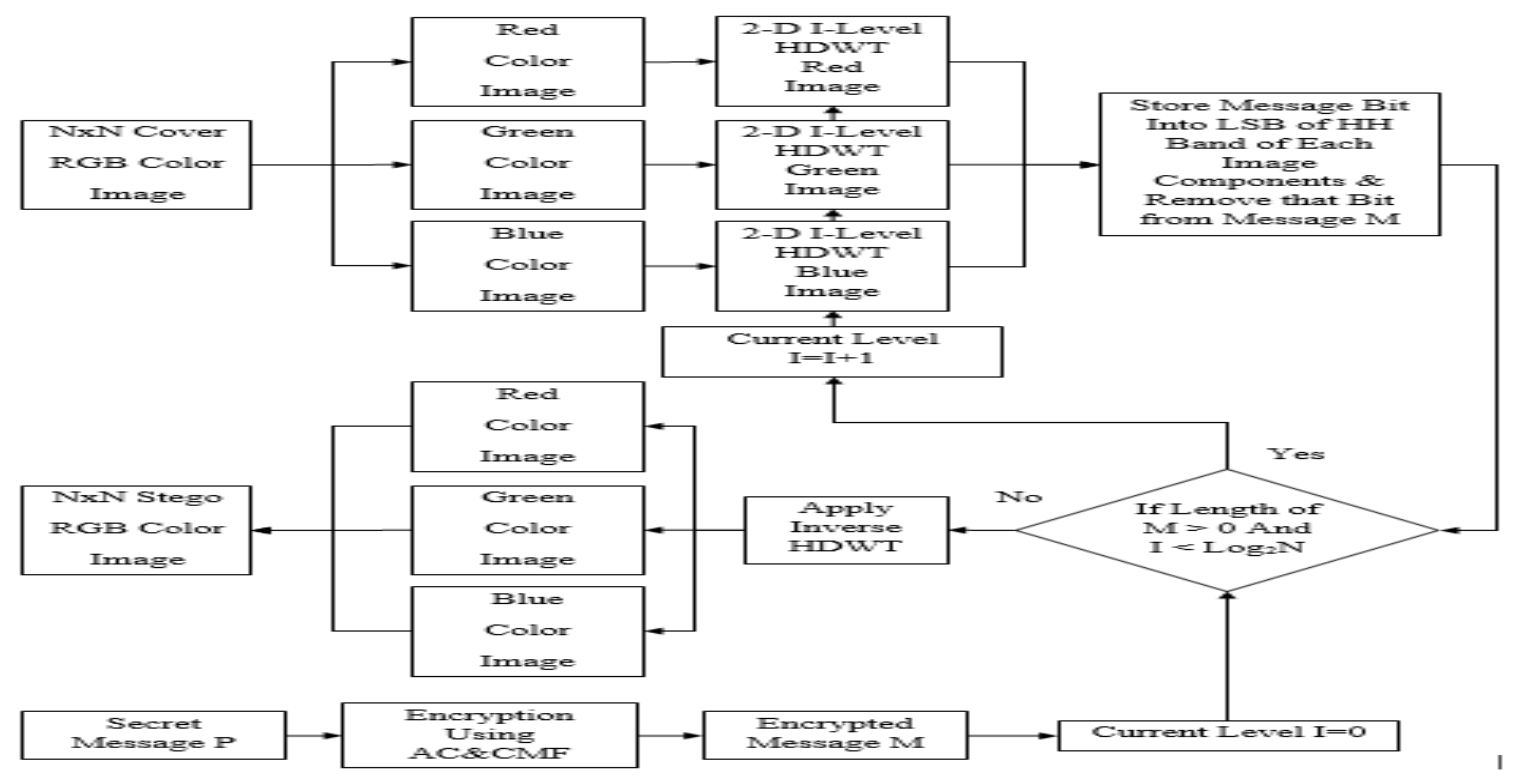

Figure 2. Embedding Procedure 


\section{B. Extraction Procedure}

Input: $A n n \times n$ stego Image.

Output: A Secret Message.

\section{Algorithm:}

\section{Steps:}

1. Read the Stego RGB Color image.

2. Separate Each Color Components from Stego Image such as R-Color Image, G-Color Image and B-Color Image.

3. Read No of Characters to be extracted and Calculate No of Bits to be extracted.

4. If no of bits to be extracted is greater than zero and current level is less than maximum level (K) then apply 2-D Haar Discrete Wavelet Transform at Next Level on each Image
Components of Stego Image otherwise go to step 7.

5. Extract Least Significant Bit (LSB) of high Frequency Coefficients of Each Image Components of Stego image and append that secret message bit to message vector.

6. Decrease no of bits to be extracted and go to step 4.

7. Prepare Message Vector.

8. Apply Decryption procedure of Modified ASCII conversion and Cyclic mathematical function based Cryptography to create original secret message for display.

9. Stop.

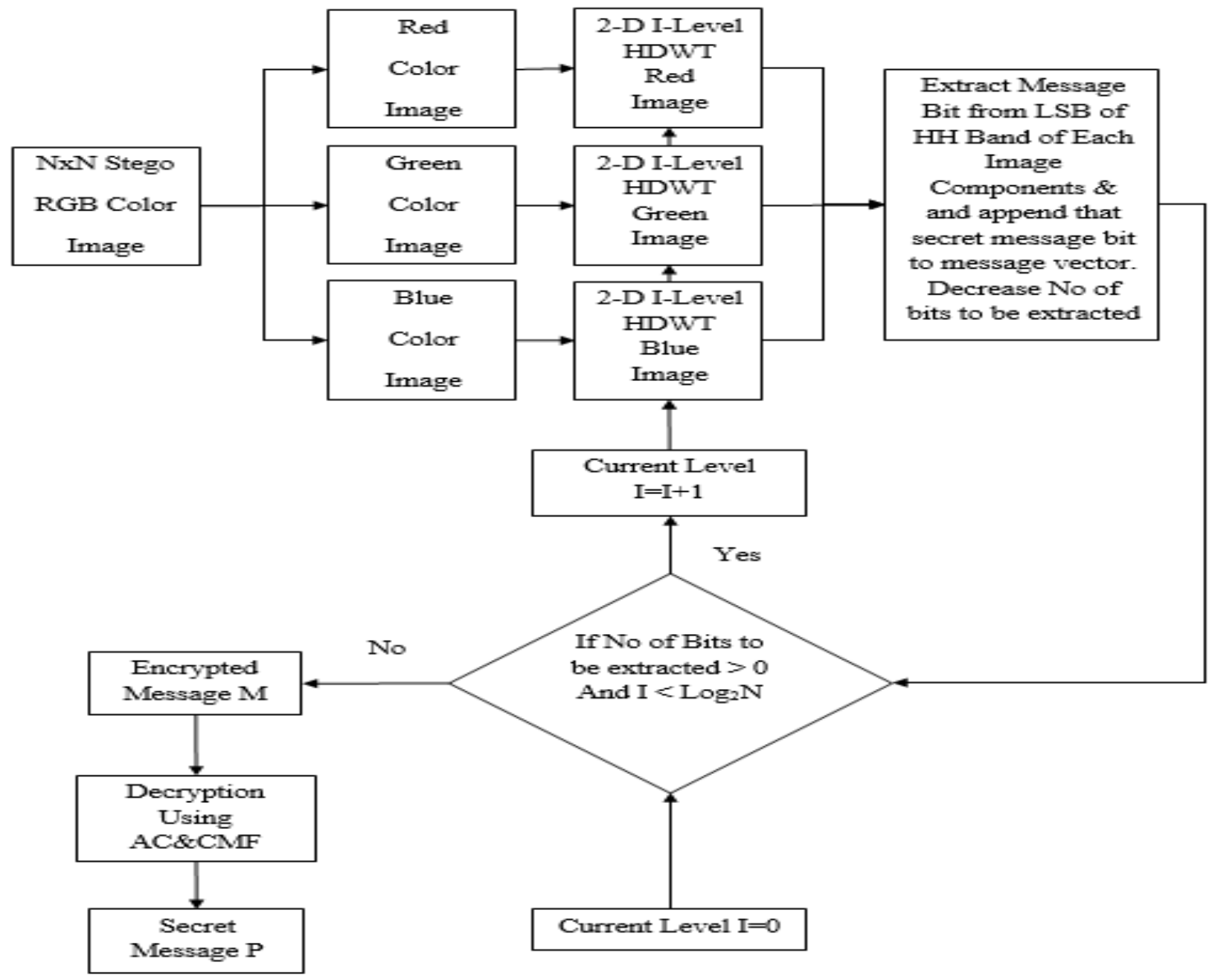

Figure 3. Extracting Procedure 


\section{EXPERIMENTAL RESULT}

This Section will investigate the performance of the proposed system with experiments. The program coded in Matlab is run on a personal computer whose operating system is Microsoft windows 7 ultimate. The CPU of the personal computer is Intel(R) Core(TM) i5 CPU M 460 @ $2.53 \mathrm{GHz}$ and its RAM is 3 GB. The Images "Lena”, “Airplane”, "Boat”, "Peppers", "Tiffany", "Gold Hill”, "Barbara", "Baboon", "St Stephens" and "Light House" are shown below which are used as a cover images.

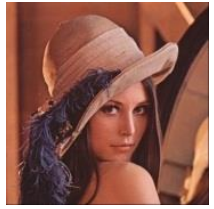

Lena

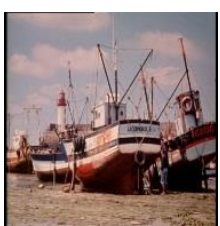

Boat

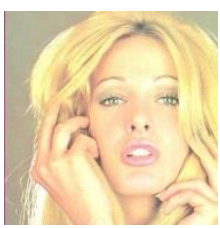

Tiffany

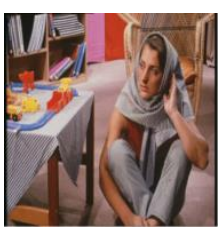

Barbara

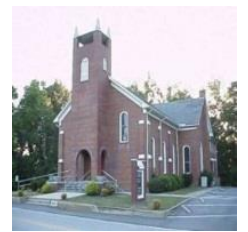

St Stephens

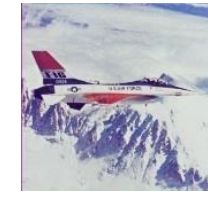

Airplane

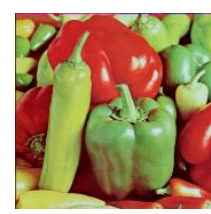

Peppers

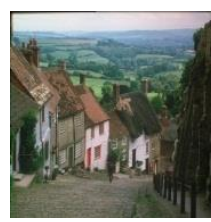

Gold Hill

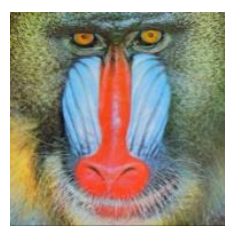

Baboon

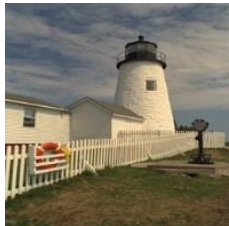

Light House
The Aim of this experiment is to find and Compare MSE and PSNR after applying multilevel 2-D HDWT using LSB and Multilevel 2-D using Alpha Blending with $\alpha=0.004$ on different size of messages for 256X256 "Lena" image.

Table 1. MSE and PSNR of Proposed Method

\begin{tabular}{|c|c|c|c|c|}
\hline $\begin{array}{c}\text { Message Size in } \\
\text { Bytes }\end{array}$ & \multicolumn{2}{|l|}{ Multilevel 2-D HDWT Using LSB } & \multicolumn{2}{c|}{$\begin{array}{c}\text { Multilevel 2-D HDWT using } \\
\text { Alpha Blending ( } \alpha=0.004)\end{array}$} \\
\cline { 2 - 5 } & MSE (\%) & PSNR (db.) & MSE (\%) & PSNR (db.) \\
\hline 1 byte & 0.000091553 & 88.5141 & 0.000091553 & 88.5141 \\
\hline 2 bytes & 0.00010681 & 87.8446 & 0.00010681 & 87.8446 \\
\hline 5 bytes & 0.00033569 & 82.8714 & 0.00038147 & 82.3162 \\
\hline 10 bytes & 0.00068665 & 79.7635 & 0.00073242 & 79.4832 \\
\hline 50 bytes & 0.003418 & 72.7931 & 0.003479 & 72.7163 \\
\hline 100 bytes & 0.0063171 & 70.1256 & 0.0071259 & 69.6024 \\
\hline 500 bytes & 0.030014 & 63.3576 & 0.036072 & 62.5591 \\
\hline 1000 bytes & 0.059479 & 60.3872 & 0.072464 & 59.5296 \\
\hline 2000 bytes & 0.12042 & 57.3237 & 0.14548 & 56.5029 \\
\hline 3000 bytes & 0.18231 & 55.5227 & 0.21829 & 54.7404 \\
\hline 4000 bytes & 0.24268 & 54.2805 & 0.29227 & 53.473 \\
\hline 5000 bytes & 0.30209 & 53.3294 & 0.36574 & 52.4991 \\
\hline 6000 bytes & 0.36324 & 52.5289 & 0.43898 & 51.7064 \\
\hline 7000 bytes & 0.50323 & 51.1131 & 0.63571 & 50.0982 \\
\hline 8000 bytes & 0.78333 & 49.1914 & 1.0483 & 47.9258 \\
\hline
\end{tabular}

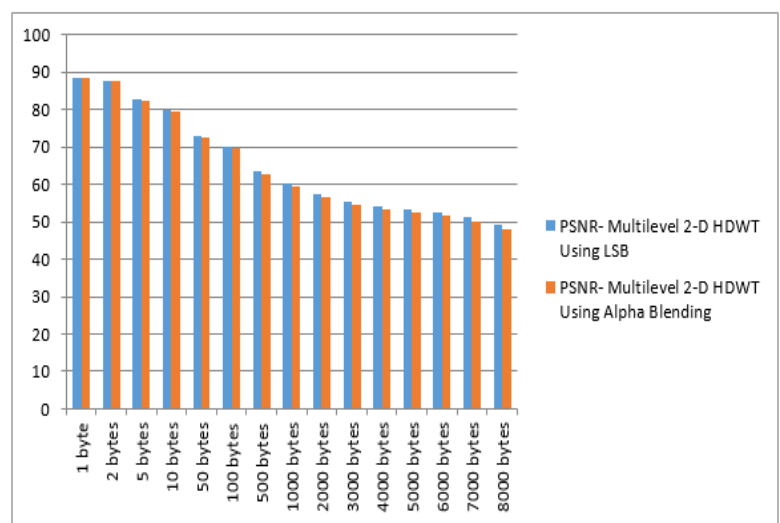

Figure 4. Graphical Representation of Proposed Work

\section{CONCLUSIONS}

From This Experiment, I conclude that multilevel 2-D HDWT using LSB gives higher PSNR value than that using Alpha Blending. Chart for comparing PSNR of 
Multilevel 2-D HDWT Using LSB and Multilevel 2-D HDWT Using Alpha Blending. Hereby it is concluded that Image Steganography Using multilevel 2-D HDWT Transform is more robust and provides more payload capacity. And cryptography based on Modified ASCII conversion and cyclic mathematical function provides high level security without increasing size of encrypted message. The security level of this method can be measured based on the PSNR value. Increase payload, PSNR value must be little decrease. Wavelet takes less time than the curvelet and provide more robustness than discrete cosine transform.

\section{REFERENCES}

[1]. Mehdi Hussain and Mureed Hussain, "A Survey of Image Steganography Techniques", International Journal of Advanced Science and Technology Vol. 54, May, 2013

[2]. Firas A. Jassim, "A Novel Steganography Algorithm for Hiding Text in Image using Five Modulus Method", International Journal of Computer Applications (0975 - 8887) Volume 72- No.17, June 2013

[3]. Chaithra H, Manjula Y, M Z Kurian, Dr.K.B.Shivakumar, Nuthan A C, "Hiding Technique Using FMM, Visual Cryptography and Genetic Algorithm", International Journal for Research and Development in Engineering (IJRDE) 2014. Vol2: Issue3

[4]. Rahul Joshi, Lokesh Gagnani, Salony Pandey, "Image Steganography with LSB", International Journal of Advanced Research in Computer Engineering \& Technology (IJARCET) Volume 2, Issue 1, January 2013

[5]. Shivani Kundra, Nishi Madaan, "A Comparative Study of Image Steganography Techniques", International Journal of Science and Research (IJSR) Volume 3 Issue 4, April 2014

[6]. Saeed Ahmed Sohag, Dr. Md. Kabirul Islam, Md. Baharul Islam, "A Novel Approach for Image Steganography Using Dynamic Substitution and Secret Key", American Journal of Engineering Research (AJER) Volume-02, Issue-09,2013
[7]. Barnali Gupta Banik, Prof. Samir K. Bandyopadhyay, "A DWT Method for Image Steganography", International Journal of Advanced Research in Computer Science and Software Engineering, Volume 3, Issue 6, June 2013

[8]. Yung-Kuan Chan, Wen -Tang Chen, Shyr-Shen $\mathrm{Yu}, \mathrm{Yu}-\mathrm{An}$ Ho, Chwei-Shyong Tsai, Yen-Ping Chu, "A HDWT -based reversible data hiding method", Elsevier Inc. 2008

[9]. Vikas pratap singh, Prof. Shrikant lade, "Haar wavelet domain analysis of image steganography", International Journal of Technical Research and Applications Volume 1, Issue 5 (Nov-Dec 2013)

[10]. Mrs.D.Mathivadhani, Dr.C.Meena, "Digital Watermarking and Information Hiding Using Wavelets, SLSB and Visual Cryptography Method", IEEE, 2010

[11]. Md. Palash Uddin, Md. Abu Marjan, Nahid Binte Sadia and Md. Rashedul Islam, "Developing a Cryptographic Algorithm Based on ASCII Conversions and a Cyclic Mathematical Function", 3rd international conference on informatics, electronics \& vision 2014

[12]. S.Shanmuga Priya, K.Mahesh, Dr.K.Kuppusamy, "Efficient Steganography Method to Implement Selected Lease Significant Bits in Spatial Domain (SLSB - SD)", International Journal of Engineering Research and Applications (IJERA)Vol. 2, Issue 3, May-Jun 2012

[13]. Mr. Vikas Tyagi, "Data Hiding in Image using least significant bit with cryptography", International Journal of Advanced Research in Computer Science and Software Engineering, Volume 2, Issue 4, April 2012

[14]. Ali Al-Ataby and Fawzi Al-Naima, "A Modified High Capacity Image Steganography Technique Based on Wavelet Transform", the International Arab Journal of Information Technology, Vol. 7, No. 4, October 2010

[15]. Prabakaran. G, Bhavani R, "A Modified Secure Digital Image Steganography Based on Discrete Wavelet Transform", International Conference on Computing, Electronics and Electrical Technologies [ICCEET], 2012 
[16]. G. Prabakaran, Dr. R. Bhavani, K Kanimozhi, "Dual Transform Based Steganography Using Wavelet Families and Statistical Methods", IEEE, 2013

[17]. Nadiya P v, B Mohammed lmran, "Image Steganography in DWT Domain using Doublestegging with RSA Encryption", International Conference on Signal Processing, Image Processing and Pattern Recognition [ICSIPR], 2013

[18]. Pratibha Sharma, Shanti Swami, "Digital Image Watermarking Using 3 level Discrete Wavelet Transform", Conference on Advances in Communication and Control Systems 2013

[19]. S.Bhargav Kumar, K.Esther Rani, "FPGA Implementation of 4-D DWT and BPS based Digital Image Watermarking", International Journal of Engineering Trends and TechnologyVolume 3 Issue2- 2012

[20]. Nikita Kashyap, G. R. SINHA, "Image Watermarking Using 3-Level Discrete Wavelet Transform (DWT)", I.J.Modern Education and Computer Science, 2012 